\title{
Correspondence
}

\section{Schizophrenia with Good and Poor Outcome}

SIR: The letter by Drs Nimgaonkar \& Murray (Journal, March 1986, 148, 343-344) misrepresents some aspects of our studies (Journal, March and April 1985, 146, 229-246 and 348-357). According to the opening and closing sentences of their letter, these studies illustrate the difficulty of obtaining longitudinal information from a cross-sectional study, and the design allowed us to do no more than confirm what is already known about the prognostic significance of certain clinical features. These comments may suggest (misleadingly) that the principal aim of our investigation was to find clinical predictors of outcome in schizophrenia. In fact, it was concerned mainly with 'cross-sectional' questions: whether patients with persisting schizophrenic symptoms also show signs indicating organic brain dysfunction (an abnormal CT scan, neurological soft signs and/or cognitive impairment); whether these 'organic' signs are inter-related; and whether they are associated with any particular chronic psychiatric symptoms. The 'longitudinal' information, imperfect because obtained retrospectively, was reported to indicate whether the groups with good and poor outcome differed in the early stages of illness, especially in the quality of remission and response to neuroleptics at that time.

The possibility that some of the 'organic' abnormalities may have been "... . consequent upon poor outcome or factors associated with it ...", was not ignored but discussed at some length in the second and third papers in the series. Though these papers presented in detail the abnormalities found and included tentative interpretations, they were somehow disregarded in the letter. Further, we have not claimed that our data "have eliminated all likelihood of pharmacological tolerance" in patients who respond unsatisfactorily to treatment with neuroleptics. We have only said that our data on drug bioavailability and prolactin response provided no evidence for such tolerance.

Finally, though our sample was not representative for the prevalence of particular types of outcome, it included the full range of outcome states, from asymptomatic remission to chronic psychosis. Drs Nimgaonkar and Murray comment disapprovingly that the non-representative character of the sample did not deter us from "making the sweeping generalisations about possible sub-types of schizophrenia". In fact, our "sweeping generalisations" consisted only in supporting the distinction between sub-types with good and poor outcome-a distinction which under various names has been discussed for at least half a century.

TAMARA KOLAKOWSKA

Department of Psychiatry Research Unit

Oxford University

Littlemore Hospital

Oxford $O X 44 X N$

Expressed Emotion and Relapse in Schizophrenia

SIR: In their letter (Journal, February 1986, 148, 215) about our recent paper on expressed emotion (EE) and relapse in schizophrenia (MacMillan et al, 1986a), Drs Leff and Vaughn are apparently undecided whether our results are an inappropriately interpreted replication of their own work or are flawed by fundamental methodological defects. We wish to clarify some of the issues involved.

The chi-squared analysis used by Leff \& Vaughn to give a positive interpretation of our results ignores the confounding effects of treatment and duration of illness preceding admission, and makes inefficient use of available data on time to relapse (Peto et al, 1977). Moreover, it is incorrect to exclude even the small numbers of patients lost to follow-up in this study. In dismissing the influence of treatment status Leff and Vaughn make the customary error (Altman,

TABLE I.

\begin{tabular}{lccc}
\hline & Active & Placebo & Total \\
\hline Low EE & 14 & 10 & 24 \\
High EE & 9 & 18 & 27 \\
\hline Total & 23 & 28 & 51 \\
\hline
\end{tabular}

$\chi^{2}=3 \cdot 21$, d.f. $=1, P>0.05$.

Low EE: odds on Active treatment: $\frac{14}{24} / \frac{10}{21}=1.4$.

High EE: odds on Active treatment: $\frac{9}{27 / \frac{18}{27}}=0.5$. 